\title{
Long-Term Consequences of Neonatal Caffeine on Ventilation, Occurrence of Apneas, and Hypercapnic Chemoreflex in Male and Female Rats
}

\author{
GASPARD MONTANDON, AIDA BAIRAM, AND RICHARD KINKEAD
}

Département de Pédiatrie, Université Laval, Centre de recherche Hôpital St-François d'Assise, Québec, Quebec, Canada G1L 3L5

\begin{abstract}
Caffeine is an adenosine receptor antagonist commonly used as a respiratory stimulant to treat neonatal apneas of premature newborn. Neonatal caffeine treatment (NCT) has long-term effects on adenosine receptor expression and distribution; however, the potential effects of NCT on respiratory control development are unknown. To address this issue, rat pups received orally each day from postnatal d 3-12, $15 \mathrm{mg} / \mathrm{kg}$ of caffeine (NCT), water (vehicle), or were undisturbed during early life (control). Measurements of resting ventilation, apnea index, and ventilatory response to moderate hypercapnia $\left(\mathrm{FiCO}_{2}=\right.$ 0.05 ) were made using whole-body plethysmography at postnatal d 20 (juvenile) and adulthood. At d 20, resting respiratory variables were not affected by the treatments. Juvenile NCT male rats showed a $22 \%$ higher minute ventilation response to hypercapnia than vehicle rats. However, oral gavage alone increased the frequency component of the response by $11 \%$. In adult males, caffeine increased the resting respiratory frequency by $15 \%$. In these animals, the tidal volume response to hypercapnia was increased by $15 \%$, whereas the frequency response was decreased by $20 \%$. In juvenile and adult females, no differences were observed between treatments. In juvenile rats of both sexes, gavage increased the apnea index by at least $200 \%$. These results show that NCT and gavage influence respiratory control during early life and that these effects persist until adulthood. The underlying mechanisms are unclear, but may be related to persistent changes in adenosinergic neurotransmission because neonatal caffeine administration increases $\mathrm{A}_{1}$ adenosine receptor density in adult rats. (Pediatr Res 59: 519-524, 2006)
\end{abstract}

$\mathrm{P}^{\mathrm{e}}$ eriodic breathing and apnea are common in neonates, especially in preterm infants (1), and caffeine administration is the treatment of choice for apnea of prematurity. The effects of caffeine are mediated by adenosine $\mathrm{A}_{1}$ and $\mathrm{A}_{2}$ receptor inactivation to prevent the actions of endogenous adenosine. Although caffeine administration in neonates can last several days, little is known about the potential long-term consequences of this treatment. In rats, NCT, administrated by gavage at dosages comparable to those used therapeutically, has long-term effects on locomotor activity (2) and cognitive

Received July 26, 2005; accepted November 11, 2005.

Correspondence: Gaspard Montandon, Centre de recherche (D0-711), Hôpital StFrançois d'Assise, M.Sc., 10 rue de l'Espinay, Québec, QC, Canada G1L 3L5; e-mail: gaspard.montandon.1@ulaval.ca.

Supported by the Foundation for the Research into Children's Disease, Canada Research Chair in Respiratory Neurobiology, and Canadian Institutes of Health Research.

DOI: 10.1203/01.pdr.0000203105.63246.8a capacities (3). These effects have been attributed to persistent changes in adenosinergic neurotransmission because chronic caffeine administration during the neonatal period increases adenosine $A_{1}$ receptor density in the CNS of adult rats (4).

In the context of respiratory regulation, many studies have investigated the possible consequences of maternal caffeine consumption during pregnancy on ventilatory control in rats; however, little attention has been directed to the persistent repercussion of caffeine treatment administrated after birth in newborn rats. Caffeine administration during gestation can modify respiratory control (5), increase the incidence of apnea in the adult animal (6), or change adenosine receptors expression in several brainstem chemosensitive sites in newborn rats (7). Nevertheless, these studies did not consider caffeinemediated changes in dam behavior that could stress the litter and thus impair CNS development in pups. These factors must be considered since disrupting mother-pup interactions-a stress that occurs during caffeine administration-affects respiratory control development $(8,9)$.

Two main questions arise from this situation. Firstly, does NCT have mid- or long-term consequences on respiratory control development and on apnea occurrence? And, secondly, does exposure to a stress, such as gavage and pup handling, alter respiratory control development? These questions were addressed in an animal model that is relevant to clinical situations. To assess the impact of NCT on respiratory control development, resting ventilation and apnea incidence were measured using whole-body plethysmography in caffeine-treated (NCT group) and nontreated (gavage; vehicle group) female and male rats at different developmental stages: postnatal d 20 (juvenile) and at adulthood. Moderate hypercapnia $\left(\mathrm{FiCO}_{2}=0.05\right)$ was used to increase arterial $\mathrm{CO}_{2}$ levels (as it occurs during asphyxia) and to reveal modifications of the hypercapnic chemoreflex. Ventilation was also measured in unhandled (control) animals, to determine whether animal handling and gavage procedure alter respiratory control development.

Abbreviations: $\mathbf{F i C O}_{2}$, fractional concentration of carbon dioxide in the inspired gas; $\mathbf{f}_{\mathbf{R}}$, respiratory frequency; NCT, neonatal caffeine treatment; $\mathbf{T}_{\mathbf{E}}$, expiratory duration; $\mathbf{T}_{\mathbf{I}}$, inspiratory duration; $\dot{V}_{\mathbf{E}}$, minute ventilation; $\dot{V} o_{2}$, oxygen consumption; $\mathbf{V}_{\mathbf{T}}$, tidal volume 


\section{METHODS}

Animal and housing conditions. Experiments were performed on 76 juvenile (postnatal d 20) and 62 adult (3-4 mo old) Sprague-Dawley rats. All animals were born in our animal care facility. Dams and males were obtained from Charles Rivers Canada (St.-Constant, QC, Canada). Rats were supplied with food and water ad libitum and maintained in standard laboratory conditions $\left(21^{\circ} \mathrm{C}, 12: 12 \mathrm{~h}\right.$ dark-light cycle: lights on at $0800 \mathrm{~h}$ and off at 2000 h). Laval University Animal Care Committee approved the experimental procedures used in this study, and the protocols were in accordance with the guidelines detailed by the Canadian Council on Animal Care.

Mating and neonatal caffeine treatment. Virgin females were mated and delivered $10-16$ pups. No litter reduction was made because mortality (10\%) related to the gavage procedure reduced the number of pups. At the end of treatment, the mean number was $10 \pm 2$ pups per litter. The NCT protocol was inspired from that of Guillet (2). Three days after delivery (P3), the NCT began and caffeine citrate $15 \mathrm{mg} / \mathrm{kg}$ (Sabex, Boucherville, QC, Canada) or water (vehicle groups) was administrated daily by gavage to pups on postnatal d 3-12 in a volume of $0.05 \mathrm{~mL} / 10 \mathrm{~g}$ body weight. A polyethylene tubing (PE10) was gently inserted into the esophagus up to the stomach, and the appropriate volume was injected slowly over a 30 -s period. Mortality was due mainly to the accidental insertion of the catheter into the trachea and injection of the solution into the lung. In preliminary experiments, plasma caffeine concentrations were determined in two litters by immunoassay (EMIT Caffeine Assay, Dade Behring, Deerfield, IL) at d 12, $1 \mathrm{~h}$ after the last gavage to assess the effectiveness of the treatment. Caffeine concentrations were also determined at postnatal $\mathrm{d} 20$ to ensure that caffeine was eliminated at the time of measurements. Control groups consisted of two litters that were not disturbed during the first $14 \mathrm{~d}$ of life.

Experimental groups. Series I tested the mid-term effects of NCT on the ventilatory activity at rest and during acute exposure to moderate hypercapnia $\left(\mathrm{FiCO}_{2}=0.05\right)$ at postnatal d 20 (juvenile rats). Series II addressed the long-term effects of NCT at adulthood. Each series involved six groups: control males (juvenile, $n=6$; adult, $n=6$ ), vehicle males (juvenile, $n=13$; adult, $n=12$ ), NCT males (juvenile, $n=17$; adult, $n=13$ ), control females (juvenile, $n=6$; adult, $n=6$ ), vehicle females (juvenile, $n=15$; adult, $n=$ 13 ), and NCT females (juvenile, $n=19$; adult, $n=12$ ).

Respiratory and metabolic measurements. Measurements of $f_{R}, V_{T}, T_{I}$, and $\mathrm{T}_{\mathrm{E}}$ in unrestrained rats were obtained by whole body flow-through plethysmograph (model PLY3223, Buxco Electronics, Sharon, CT) according to our standard method $(8,10)$. In juvenile rats, rectal temperature was measured before resting measurements and after hypercapnia using a thermocouple for small rodents. In adults, core body temperature was measured continuously by telemetry. Transponders (E-mitter, Mini Mitter, Bend, Oregon) were surgically implanted 1 wk before measurements. Rats were anesthetized with ketamine/xylazine (i.p. $10 / 50 \mathrm{mg} / \mathrm{kg}$ ), the transponder was placed inside the peritoneum and was sutured behind the internal wall of the cavity. Postsurgical care consisted of three subcutaneous injections of antiinflammatory (ketoprofen, $2 \mathrm{mg} / \mathrm{kg}$ ): immediately after surgery, $24 \mathrm{~h}$, and $48 \mathrm{~h}$ postoperatively. Data obtained from adult animals compared well with results obtained in our laboratory in unoperated animals (10), thereby indicating that surgical stress did not affect our results. Barometric pressure, flow rate, chamber temperature, and humidity were also measured to express $\mathrm{V}_{\mathrm{T}}$ in milliliters per $100 \mathrm{~g}$ of body weight $(11,12)$. Composition of the gas mixtures flowing in and out of the chamber was analyzed with an oxygen analyzer (model S-3A, Ametek, Pittsburgh, PA) for subsequent calculation of oxygen consumption $\left(\mathrm{V}_{2}\right)$ with an open system (13).

Respiratory measurement protocol. For each measurement session, rats were acclimatized to the plethysmography chamber for $1 \mathrm{~h}$. Resting respiratory and metabolic measurements were made when the animal was quiet but awake and breathing room air using a data acquisition software (IOX, EMKA Technologies, Falls Church, VA). Acceptation/rejection of individual breaths was performed automatically by the software. Experience has showed us that the software's default values for most parameters are adequate to reject signals related to movement artifacts. However, sniffing-related signals were excluded by setting the $T_{I}$ rejection threshold above $120 \mathrm{~ms}$. The validity of this criteria was confirmed for each animal by comparing accepted/rejected breaths while observing the rat's behavior; the rejection threshold was adjusted if necessary. With this approach, the acceptance/rejection rate when the animal became calm and produced a steady signal was typically above $75 \%$. A similar acceptance rate or higher was observed during hypercapnia. After 15 min of normoxic measurements, a hypercapnic gas mixture was delivered to the chamber for $20 \mathrm{~min}$. In each series, animals were exposed to only one respiratory stimulus. All measurements were made between 0900 and $1300 \mathrm{~h}$.

Data and statistical analysis. "Resting" measurements of ventilatory variables were obtained using DataAnalyst software (EMKA Technologies) by averaging $10 \mathrm{~min}$ of stable recording, whereas a 6-min average was taken for each variable from the 14th to the 20th min of hypercapnic exposure. The hypercapnic ventilatory response was expressed as a percentage change of the hypercapnic mean relative to mean baseline values. A value of $0 \%$ signifies that there was no change due to hypercapnia, whereas a value of $100 \%$ indicates a 2 -fold increased relative to baseline value.

The apnea index was determined by counting the number of apneas per hour. Based on the criteria of Mendelson and colleagues (14), an apnea was defined as an absence of flow for at least two normal breathing cycles. Thus, interruption of airflow had to exceed $1 \mathrm{~s}$ in juveniles and $2 \mathrm{~s}$ in adults to qualify as an apnea. Two types of apneic pauses were observed: spontaneous and post-sigh apneas. A spontaneous apnea was characterized by an interruption of flow, whereas a post-sigh apnea was preceded by a breath with an amplitude twice the resting tidal volume.

Data were analyzed using a one-way ANOVA (JMP 5.1, SAS Institute Inc., Cary, NC) for both sexes at each age. Responses were compared using a two-way ANOVA for repeated measures (hypercapnic stimulus $\times$ treatment). These analyses were followed by post hoc tests when appropriated. However, three-way ANOVA were used to assess treatment effects across stage groups (hypercapnic stimulus $\times$ treatment $\times$ age) and sex specificity (hypercapnic stimulus $X$ treatment $\times$ sex). Interaction tests were used to show sex- or age-related differences in the treatment effects (15). Caffeine dosages were compared by a $t$ test. Data were considered statistically different when $p<0.05$. Data are expressed using means $\pm 1 \mathrm{SD}$ according to the guidelines proposed by the American Physiologic Society (16).

\section{RESULTS}

Plasma caffeine concentrations. At P12, mean plasmatic caffeine concentration was $13 \pm 3 \mathrm{mg} / \mathrm{L}(n=7)$ in the NCT group, whereas caffeine was not detected in vehicle group $(<5$ $\mathrm{mg} / \mathrm{L} ; n=8$ ). In the neonatal unit, caffeine levels measured in infants typically range between 8 and $20 \mathrm{mg} / \mathrm{L}$. At P20, $8 \mathrm{~d}$ after the last caffeine administration, no caffeine was detected in samples from vehicle and NCT rats $(n=10$ for each group).

Series I: resting ventilation in juvenile rats. Baseline ventilatory measurements of vehicle juvenile animals (Table 1) were comparable to those reported previously for Sprague-Dawley rats of this age group [e.g. (17)]. In vehicle juvenile rats, no differences were found between males and females in all variables and no changes were observed in resting values between NCT and vehicle groups. Furthermore, NCT did not affect body weight in male and female rats.

Series I: hypercapnic ventilatory response in juvenile rats. For both sexes and conditions, hypercapnia elicited significant changes in all ventilatory variables. No differences in hypercapnic ventilatory responses were found between males and females (sex effect: $p>0.05$ for all variables). Comparisons of ventilatory measurements between treatments and sexes are presented in Fig. 1. In juvenile males, $\dot{V}_{E}$ response to hypercapnia was $22 \%$ greater in NCT than in vehicle (treatment effect: $p=0.047$ ), owing in part to a greater $\mathrm{V}_{\mathrm{T}}$ response $(12 \%, p=0.023)$. Moreover, $\dot{V}_{E}$ response was $36 \%$ greater in NCT than in control (undisturbed animals) $(p=0.020)$, owing in part to a $14 \%$ greater $\mathrm{f}_{\mathrm{R}}$ response $(p=0.008)$. Gavage alone increased the frequency response by $11 \%$ in vehicle rats relative to controls $(p=0.037)$. Moreover, the $\mathrm{T}_{\mathrm{E}}$ response to hypercapnia was higher in vehicle $(8 \%)$ and NCT animals (8\%) than in controls ( $p=0.025$ and $p=0.018$, respectively), indicating that the gavage procedure per se modified the hypercapnic ventilatory response. $\dot{V}_{2}$ during hypercapnia was unchanged by NCT and gavage procedure in both sexes. In juvenile females, neither NCT nor gavage affected the hypercapnic ventilatory response. 
Table 1. Effects of NCT on resting ventilatory variables of juvenile male and female rats: Series I

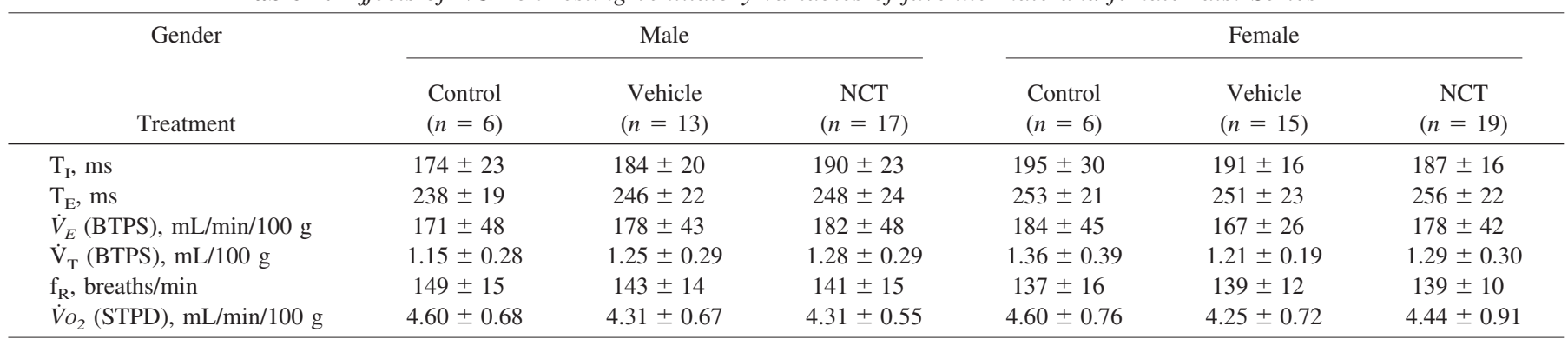

Values are expressed as means \pm SD. Resting values were obtained in quiet but awake rats $\geq 1 \mathrm{~h}$ after the animal acclimated to the plethysmographic chamber. STPD, standard temperature pressure, dry $\left(0^{\circ} \mathrm{C}, 760 \mathrm{~mm} \mathrm{Hg}\right)$; BTPS, body temperature and pressure, saturated with water vapor.

Series II: resting ventilation in adult rats. Baseline ventilatory measurements obtained in adult controls (Table 2) were comparable to those reported in other studies using the same rat strain $[$ e.g. (8)]. In control animals, no differences were observed between females and males. In NCT animals, however, $\mathrm{V}_{\mathrm{T}}$ of females was $56 \%$ higher than in males (sex effect: $p=0.002$ ), whereas $\mathrm{f}_{\mathrm{R}}$ was lower by $22 \%$ in NCT females $(p=0.0005)$.

In male rats, NCT reduced $\mathrm{T}_{\mathrm{E}}$ by $15 \%$ (treatment effect: $p$ $=0.011)$ when compared with vehicle animals, and consequently increased $\mathrm{f}_{\mathrm{R}}$ by $15 \%(p=0.004)$. However, NCT had no net effect on resting $\dot{V}_{E}$. In female rats, no differences were found between vehicle and NCT animals for these variables. In males, comparisons between control and vehicle rats indicated that the gavage procedure tended to reduce $\mathrm{f}_{\mathrm{R}}$ by $9 \%(p$ $=0.08)$, but increased $\dot{V}_{O_{2}}$ by $20 \%(p=0.006$; Table 2$)$. In females, gavage procedure increased resting $\mathrm{T}_{\mathrm{I}}$ by $20 \%$ ( $p=$ $0.017)$. No influence of sex on treatment effects was observed for $\mathrm{f}_{\mathrm{R}}, \mathrm{V}_{\mathrm{T}}$, and $\dot{V}_{E}$ (interaction tests for sex $\times$ treatment: $p=$ $0.11, p=0.09$, and $p=0.94$, respectively).

Series II: hypercapnic ventilatory response in adult rats. Figure 2 shows that, in adult males, NCT increased $\mathrm{V}_{\mathrm{T}}$ response by $15 \%$ (treatment effect: $p=0.002$ ), whereas it decreased breathing frequency response by $20 \%(p=0.0006)$ and $\mathrm{T}_{\mathrm{I}}$ response by $9 \%(p=0.008)$. Unlike juveniles, however, no differences in the hypercapnic ventilatory response were observed between vehicle and control animals. Only a slight increase in $\mathrm{f}_{\mathrm{R}}$ response was observed in male rats $(13 \%$, $p=0.055)$. Gavage did not affect the hypercapnic ventilatory response of other variables at adulthood. Finally, NCT had no effect on the ventilatory responses of adult females. By testing the interaction between sex, treatment, and hypercapnia, NCT decreased the $f_{R}$ response by $23 \pm 8 \%$ in adult males, whereas a small but not significant increase was observed in females (difference between male and female $=30.36 \%$; interaction test: $p=0.025$ ). For $\mathrm{V}_{\mathrm{T}}$ and $\dot{V}_{E}$, no sex specificity was observed.

Comparing responses between juvenile and adult vehicle males revealed a $13 \%$ increase in the $\dot{V}_{E}$ response during development (age effect: $p=0.046$ ), owing mainly to a $21 \%$ increase in the $\mathrm{f}_{\mathrm{R}}$ response $(p<0.0001)$ inasmuch as the $\mathrm{V}_{\mathrm{T}}$ response decreased by $12 \%(p=0.002)$. In NCT male rats, no change was observed in these variables during maturation. However, age did not affect treatment influences for $f_{R}, V_{T}$, and $\dot{V}_{E}$ for both sexes.
Apnea index at rest. Spontaneous apneas were observed in all groups of rats except in control adults (Fig. 3B). In vehicle and NCT rats of both sexes, the apnea index decreased strongly during maturation (age effect: $p<0.05$ in all groups). In juvenile males, both treatments (vehicle and NCT) increased apnea index in comparison with controls (treatment effect: $p=0.047$ and $p=0.013$, respectively). In juvenile females, NCT tended to increase the apnea index; however, only the index measured in vehicle rats was statistically higher than that of controls $(p=0.025)$. These results show that gavage increased the occurrence of apneas in juvenile, whereas it had no effect on spontaneous apnea in adults.

Conversely, quantification of post-sigh apneas showed that, in males, neither age nor gavage affect the apnea index (Fig. 4B). In females, however, the apnea index of adult vehicle rats was $54 \%$ less than that of juvenile rats (age effect: $p=0.0003$ ). Moreover, comparing vehicle to NCT in juvenile females showed that caffeine diminished the post-sigh apnea index by 38\% (treatment effect: $p=0.010$ ).

\section{DISCUSSION}

Neonatal caffeine administration is commonly used to treat respiratory disorders in newborn, especially in preterm infants. The use of pharmacological agents during a critical period of development is always a matter of concern given

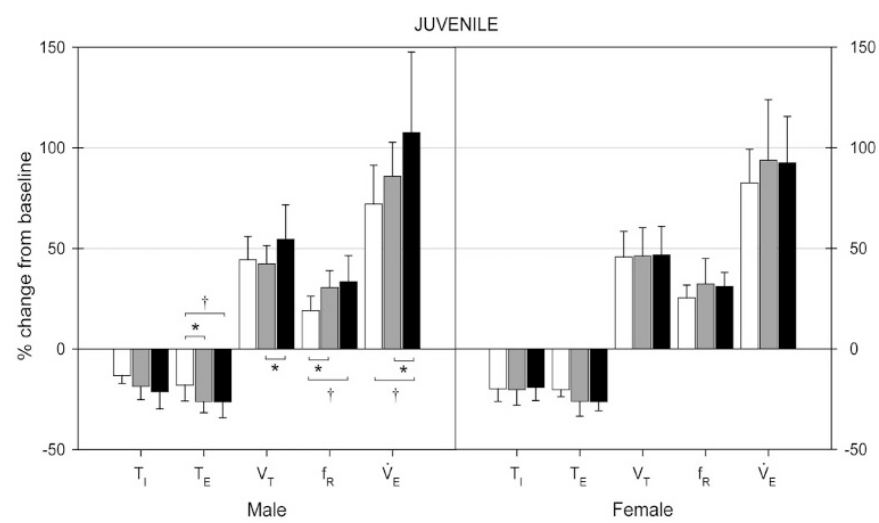

Figure 1. Comparison of the ventilatory responses to moderate hypercapnia $\left(\mathrm{FiCO}_{2}=0.05\right)$ between control (white bars), vehicle (gray bars), and NCT treated rats (black bars) expressed in percentage of resting value for male ( $n=6$ in control, $n=13$ in vehicle, and $n=17$ in NCT) and female $(n=$ 6 in control, $n=15$ in vehicle, and $n=19$ in NCT) juvenile rats. Data are expressed as means $\pm \mathrm{SD}$. ${ }^{*} p<0.05$ vs vehicle. $\dagger p<0.05$ vs control. 
Table 2. Effects of NCT on resting ventilatory variables of adult male and female rats: Series II

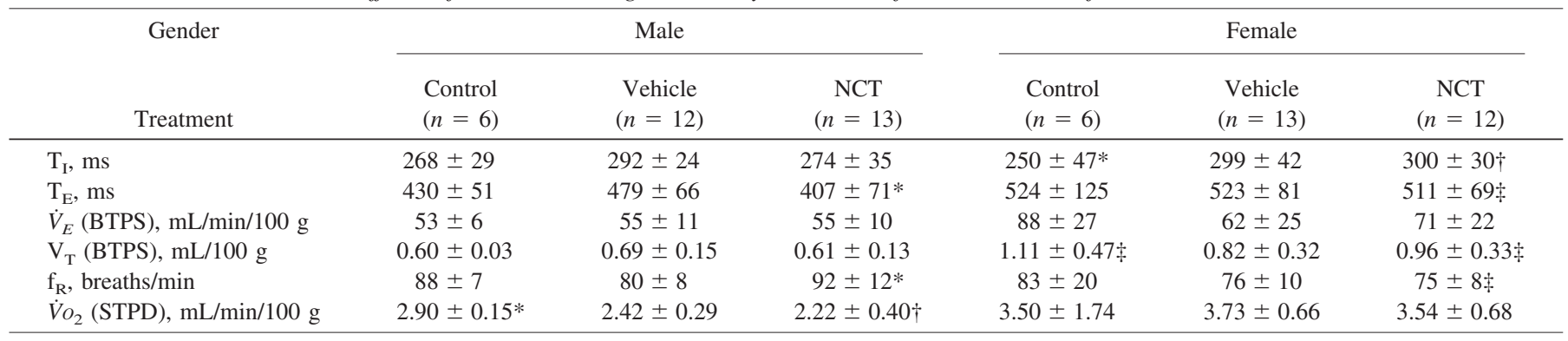

Value are means \pm SD. Resting values were obtained in quiet but awake rats $\geq 1 \mathrm{~h}$ after the animal acclimated to the plethysmographic chamber. $* p<0.05$ vs vehicle. $\dagger p<0.05$ vs control. $\ddagger p<0.05$ vs male.

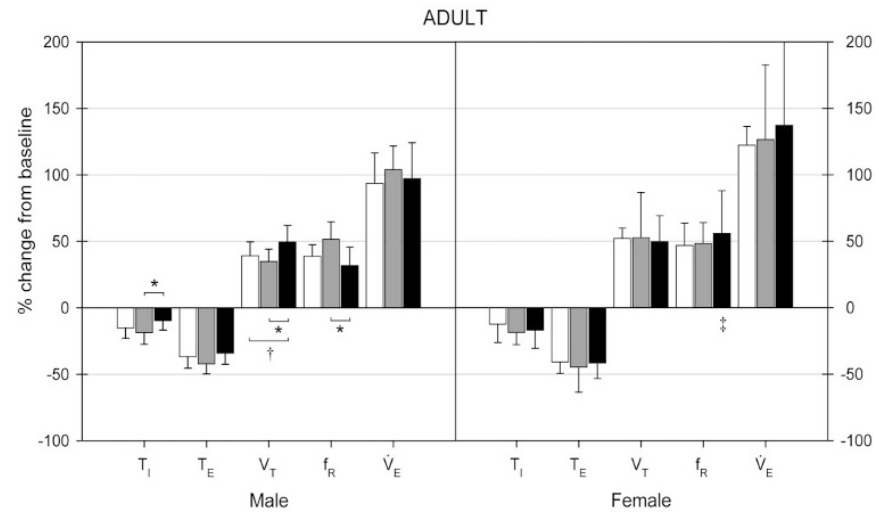

Figure 2. Comparison of the ventilatory responses to moderate hypercapnia $\left(\mathrm{FiCO}_{2}=0.05\right)$ expressed in percentage of resting value for male $(n=6$ in control, $n=12$ in vehicle, and $n=13$ in NCT) and female ( $n=6$ in control, $n=13$ in vehicle, and $n=12$ in NCT) adult rats. White (control), gray (vehicle), and black (NCT) bars shows means \pm SD. ${ }^{*} p<0.05 v s$ vehicle. $\dagger p$ $<0.05$ vs control. $\ddagger p<0.05$ vs males.

their potential impact on CNS maturation and subsequent functions. Several studies have addressed the effects of perinatal caffeine administration on CNS performance under various conditions. However, to the best of our knowledge, this study is the first to use an animal model to address the potential consequence of caffeine on CNS development in a context that (besides the accidental 10\% mortality rate due to gavage) mimics the clinical situation relatively well, i.e. neonatal administration with a focus on respiratory control development. Our results suggest that NCT exerts specific effects on respiratory control in male juvenile rats that change and subside partially with maturation. Furthermore, our data show that pup manipulation and gavage alone may contribute to these effects on respiratory control, thus emphasizing the importance of performing proper sham and control experiments especially when interventions are performed during early life. Although mainly descriptive in nature, this study nonetheless raises important question concerning the impact of disruptions of mother-pup interactions and caffeine administration on respiratory control, especially in juvenile animals.

NCT-related changes in juvenile rats. In male rats, NCT increases the magnitude of the $\dot{V}_{E}$ response to hypercapnia by $22 \%$ without modifying resting ventilation. Because $\dot{V}_{2}$ is not altered by caffeine, it is unlikely that these changes are related to metabolism, and suggest that $\mathrm{NCT}$ increases $\mathrm{CO}_{2}$ chemosensitivity. The mechanisms responsible for this increase in
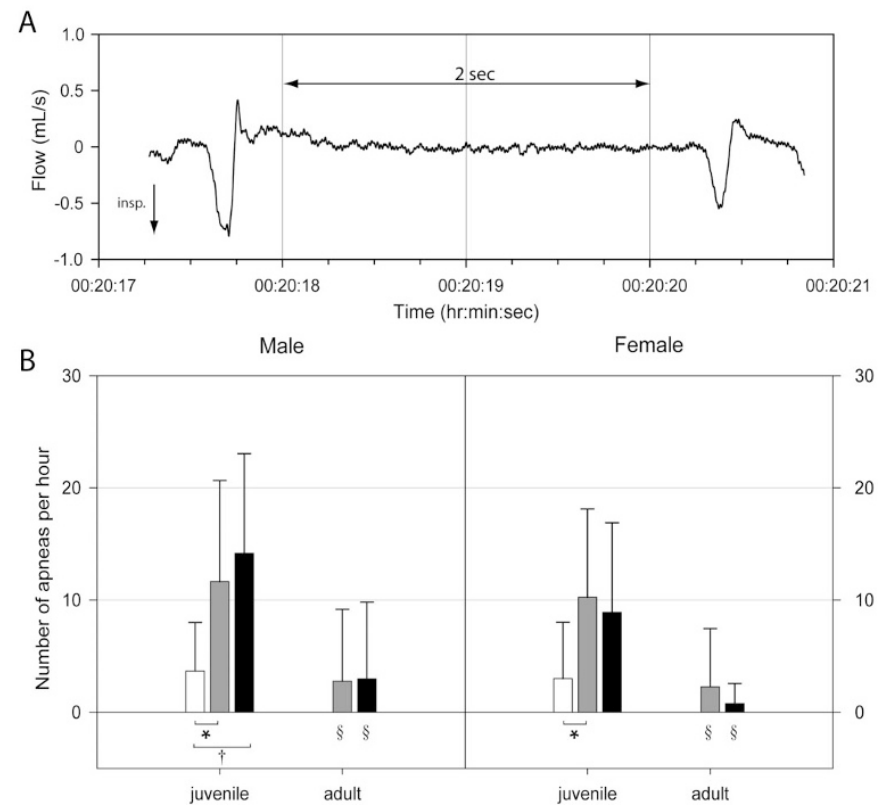

Figure 3. Incidence of spontaneous apneas at rest in juvenile (P20) and adult male and female rats. (A) Sample of plethysmographic signal representing a spontaneous apnea of more than $2 \mathrm{~s}$ of adult rat. Downward deflection represents inspiration. $(B)$ Mean numbers of apneas per hour of rats at rest. White (control), gray (vehicle), and black (NCT) bars shows means \pm SD. *p $<0.05 v s$ vehicle. $\dagger p<0.05$ vs control. $\$ p<0.05 v s$ juveniles. In juvenile males: $n=6$ in control, $n=13$ in vehicle, and $n=17$ in NCT. In juvenile females: $n=6$ in control, $n=15$ in vehicle, and $n=19$ in NCT. In adult males, $n=6$ in control, $n=12$ in vehicle, and $n=13$ in NCT. In adult females, $n=6$ in control, $n=13$ in vehicle, and $n=12$ in NCT.

chemosensitivity are unknown and our data do not allow us to determine where these effects occur (CNS versus carotid body). It is known, however, that in utero caffeine exposure increases adenosine $A_{1}$ receptor expression in the brainstem of neonatal rats (7), and that NCT increases adenosine $\mathrm{A}_{1}$ receptor expression in 28-d-old rats (19). It has been proposed that when caffeine occupies the adenosine receptor's binding site, the number of receptors increases to maintain the efficiency of adenosinergic systems (20). The fact that adenosine receptor activation by the nonspecific agonist phenyl isopropyl adenosine enhances the hypercapnic ventilatory response of rats (21) is consistent with the hypothesis that NCT-related increase in adenosine receptor expression contributes (at least in part) to enhancement of the hypercapnic ventilatory response in male rat pups. 
A

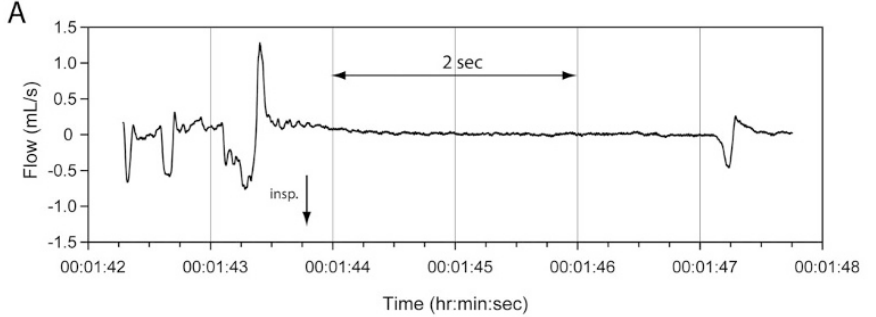

B

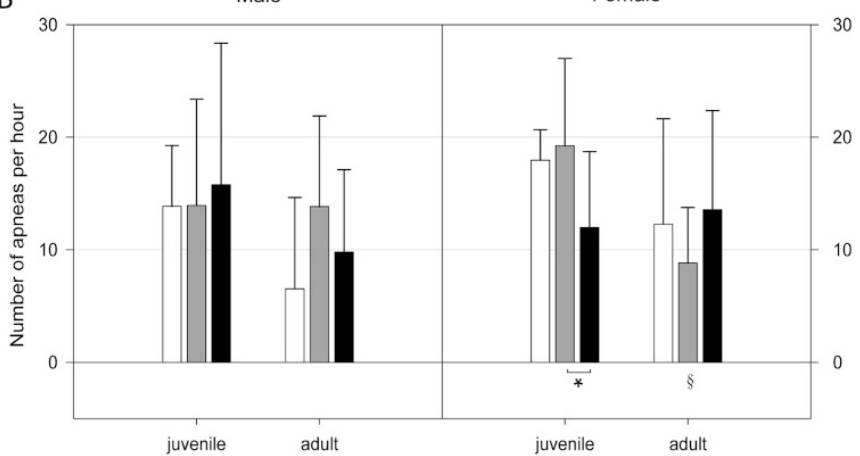

Figure 4. Incidence of post-sigh apneas at rest in juvenile (P20) and adult male and female rats. (A) Sample of plethysmographic signal representing a sigh followed by an apnea of more than $2 \mathrm{~s}$ of adult rat. Downward deflection represents inspiration. $(B)$ Mean numbers of apneas per hour of rats at rest. White (control), gray (vehicle), and black (NCT) bars shows means \pm SD. * $p$ $<0.05 v s$ vehicle. $\$ p<0.05 v s$ juveniles. In juvenile males: $n=6$ in control, $n=13$ in vehicle, and $n=17$ in NCT. In juvenile females: $n=6$ in control, $n=15$ in vehicle, and $n=19$ in NCT. In adult males, $n=6$ in control, $n=$ 12 in vehicle, and $n=13$ in NCT. In adult females, $n=6$ in control, $n=13$ in vehicle, and $n=12$ in NCT.

NCT-related changes in adult rats. The increase in $f_{R}$ observed in NCT versus vehicle rats was surprising but consistent with studies showing that caffeine administration has long-term consequences on behavior $(2,3)$ owing to changes in adenosine receptor distribution and affinity $(4,22)$. Accordingly, long-term caffeine treatment results in dose-dependent increases in adenosine $A_{1}$ receptor agonist binding sites in the CNS of adult rats $(20,23)$. However, because of the structurespecific effects of caffeine on adenosine receptors (24), only specific brainstem structures may be changed by caffeine administration.

The pattern of the hypercapnic ventilatory response is also modified by NCT in adult males. Several studies have examined the consequences of caffeine exposure on respiratory activity of neonatal rats $(5,7)$, and results suggest that $\mathrm{CO}_{2}$ chemosensitivity is altered at the CNS level. If the same alterations persist throughout life, they may enhance central $\mathrm{CO}_{2}$ sensitivity similarly in adults. However, an increase in carotid body afferent input should not be excluded inasmuch as adenosine modulates $\mathrm{CO}_{2}$ chemosensitivity in these organs (25).

Consequences of stress related to the gavage procedure. Despite its short duration $(\sim 30 \mathrm{~s})$, the gavage procedure is a form of stress that is repeatedly imposed upon the animal during a critical period of development. In addition to the physical stress and pain caused by the gavage, this procedure disrupts mother-pup interactions also as pups had to be handled (10-15 min per day) and immobilized to administrate the treatment. This causes a brief interlude in the routine of mother-pup interactions, and alters the behavior of the mother toward its offspring (26), which results in a decreased responsiveness to stress in adult offspring. In contrast, prolonged maternal separation $(3 \mathrm{~h} / \mathrm{d}$; P3 to P12) results in sex-specific (male only) increase in the hypoxic ventilatory response $(8,9)$, whereas it decreased hypercapnic ventilatory response ( $R$. Kinkead, unpublished data). However, the gavage procedure, which combines both neonatal handling and short maternal separation $(<1 \mathrm{~h} / \mathrm{d}$ for the entire litter) increases both the hypercapnic ventilatory response and the spontaneous apnea index in juvenile male rats. Taken together, these findings suggest that the effects of the gavage procedure on ventilatory control are opposite to those observed in adult male rats previously subjected to neonatal maternal separation. The effects of neonatal handling alone (i.e. without caffeine administration) on respiratory control development has not been addressed. However, these side effects of gavage subside during the maturation as they are no longer observed in the adult, suggesting that rats can recover (at least partially) from such stress.

Apnea index changes. Spontaneous and post-sigh apneas are thought to originate from two distinct mechanisms: spontaneous apneas are related to a sudden cessation of the respiratory command at the CNS level, whereas post-sigh apneas are thought to be related to pulmonary stretch reflexes occurring after the sigh (14). In the present study, gavage increased the spontaneous apnea index in juvenile males and females but not in adults. In humans, central apneas can be provoked by several factors, including a decrease in $\mathrm{PaCO}_{2}$ below apneic threshold (27). In newborns, the eupneic threshold is very close to $\mathrm{CO}_{2}$ apneic threshold (28) and thus, minor changes in $\dot{V}_{E}$ are more likely to provoke apneas. The fact that apneas were no longer observed in adults indicates that maturational changes occur until adulthood. However, this effect was not observed for post-sigh apnea for which the index remained stable during development.

Sex specificity. In our results, NCT modified the hypercapnic ventilatory responses of juvenile and adult males. However, a sex-specific effect was observed in adult animals only, where NCT increased the $f_{R}$ response to hypercapnia of males, but not of females. Other developmental studies suggest that consequences of neonatal maternal separation (8) or caffeine treatment (3) on development are sex specific (male only). In juvenile rats, only males were affected by treatments whereas in adults, the $\mathrm{f}_{\mathrm{R}}$ response to hypercapnia of males and females was affected by NCT in an opposite manner. While sexspecific effects are often observed in developmental studies, the underlying neuroendocrine mechanisms are not fully understood but may be related to the effects of sex steroids such as testosterone and estrogens. Furthermore, in adult females, steroid hormones may have a protective effect against stress and treatment, since NCT does not alter the hypercapnic ventilatory response.

Perspectives. In summary, our results suggest that NCT exerts specific effects on respiratory control in juvenile male rats that seem to change but persist with maturation. Our data show that pup manipulation and gavage contribute to alter 
respiratory control development, thus emphasizing the importance of completing proper sham and control experiments especially when interventions are performed during early life. While it is difficult to accurately translate developmental sequences across species, data suggest that at birth the CNS of a rat is immature and may compare with that of a premature baby (29). With that in mind, the demonstration that NCT increases the hypercapnic ventilatory response and the apnea index is intriguing since in humans, enhanced sensitivity to $\mathrm{CO}_{2}$ may predispose some patients with heart failure to the development of central sleep apnea (30). Such enhanced responsiveness to hypercapnic stress might be taken seriously in young infant, especially during sleep, for which an increased sensitivity to hypercapnia could predispose to respiratory disorders.

Acknowledgments. The authors thank Dr. R. Gulemetova. We also thank Evelyne Vachon and Sylvie Viger (animal care specialists) for their excellent work with the gavage procedures and maintenance of our animal colony.

\section{REFERENCES}

1. Al-Matary A, Kutbi I, Qurashi M, Khalil M, Alvaro R, Kwiatkowski K, Cates D, Rigatto H 2004 Increased peripheral chemoreceptor activity may be critical in destabilizing breathing in neonates. Semin Perinatol 28:264-272

2. Guillet R 1990 Neonatal caffeine exposure alters adenosine receptor control of locomotor activity in the developing rat. Dev Pharmacol Ther 15:94-100

3. Fisher S, Guillet R 1997 Neonatal caffeine alters passive avoidance retention in rats in an age- and gender-related manner. Brain Res Dev Brain Res 98:145-149

4. Guillet R, Kellogg C 1991 Neonatal exposure to therapeutic caffeine alters the ontogeny of adenosine A1 receptors in brain of rats. Neuropharmacology 30:489496

5. Bodineau L, Cayetanot F, Sadani-Makki F, Bach V, Gros F, Lebleu A, Collin T, Frugiere A 2003 Consequences of in utero caffeine exposure on respiratory output in normoxic and hypoxic conditions and related changes of Fos expression: a study on brainstem-spinal cord preparations isolated from newborn rats. Pediatr Res 53:266-273

6. Tye K, Pollard I, Karlsson L, Scheibner V, Tye G 1993 Caffeine exposure in utero increases the incidence of apnea in adult rats. Reprod Toxicol 7:449-452

7. Herlenius E, Aden U, Tang LQ, Lagercrantz H 2002 Perinatal respiratory control and its modulation by adenosine and caffeine in the rat. Pediatr Res 51:4-12

8. Genest SE, Gulemetova R, Laforest S, Drolet G, Kinkead R 2004 Neonatal maternal separation and sex-specific plasticity of the hypoxic ventilatory response in awake rat. J Physiol 554:543-57
9. Kinkead R, Genest SE, Gulemetova R, Lajeunesse Y, Laforest S, Drolet G, Bairam A 2005 Neonatal maternal separation and early life programming of the hypoxic ventilatory response in rats. Respir Physiol Neurobiol 149:313-324

10. Kinkead R, Dupenloup L, Valois N, Gulemetova R 2001 Stress-induced attenuation of the hypercapnic ventilatory response in awake rats. J Appl Physiol 90:1729-1735

11. Drorbaugh JE, Fenn WO 1955 A barometric method for measuring ventilation in newborn infants. Pediatrics 16:81-87

12. Mortola JP, Frappell PB 1998 On the barometric method for measurements of ventilation, and its use in small animals. Can J Physiol Pharmacol 76:937-944

13. Mortola JP, Dotta A 1992 Effects of hypoxia and ambient temperature on gaseous metabolism of newborn rats. Am J Physiol 263:R267-R272

14. Mendelson WB, Martin JV, Perlis M, Giesen H, Wagner R, Rapoport SI 1988 Periodic cessation of respiratory effort during sleep in adult rats. Physiol Behav 43:229-234

15. Altman DG, Bland JM 2003 Interaction revisited: the difference between two estimates. BMJ 326:219

16. Curran-Everett D, Benos DJ 2004 Guidelines for reporting statistics in journals published by the American Physiological Society. Am J Physiol Regul Integr Comp Physiol 287:R247-R249

17. Doan VD, Gagnon S, Joseph V 2004 Prenatal blockade of estradiol synthesis impairs respiratory and metabolic responses to hypoxia in newborn and adult rats. Am J Physiol Regul Integr Comp Physiol 287:R612-R618

18. Joseph V, Soliz J, Pequignot J, Sempore B, Cottet-Emard JM, Dalmaz Y, Favier R, Spielvogel H, Pequignot JM 2000 Gender differentiation of the chemoreflex during growth at high altitude: functional and neurochemical studies. Am J Physiol Regul Integr Comp Physiol 278:R806-R816

19. Etzel BA, Guillet R 1994 Effects of neonatal exposure to caffeine on adenosine A1 receptor ontogeny using autoradiography. Brain Res Dev Brain Res 82:223-230

20. Boulenger JP, Patel J, Post RM, Parma AM, Marangos PJ 1983 Chronic caffeine consumption increases the number of brain adenosine receptors. Life Sci 32:11351142

21. Burr D, Sinclair JD 1988 The effect of adenosine on respiratory chemosensitivity in the awake rat. Respir Physiol 72:47-57

22. Guillet R, Kellogg CK 1991 Neonatal caffeine exposure alters developmental sensitivity to adenosine receptor ligands. Pharmacol Biochem Behav 40:811-817

23. Johansson B, Georgiev V, Lindstrom K, Fredholm BB 1997 A1 and A2A adenosine receptors and A1 mRNA in mouse brain: effect of long-term caffeine treatment Brain Res 762:153-164

24. Aden U, Herlenius E, Tang LQ, Fredholm BB 2000 Maternal caffeine intake has minor effects on adenosine receptor ontogeny in the rat brain. Pediatr Res 48:177183

25. Kumar P, Conway AF, Vandier C, Marshall NJ, Bruynseels J, Matthews GM 2000 Effect of adenosine on $\mathrm{CO} 2$ chemosensitivity. Functional, cellular, and molecular studies. Adv Exp Med Biol 475:405-410

26. Liu D, Diorio J, Day JC, Francis DD, Meaney MJ 2000 Maternal care, hippocampal synaptogenesis and cognitive development in rats. Nat Neurosci 3:799-806

27. Leevers AM, Simon PM, Dempsey JA 1994 Apnea after normocapnic mechanical ventilation during NREM sleep. J Appl Physiol 77:2079-2085

28. Khan A, Qurashi M, Kwiatkowski K, Cates D, Rigatto H 2005 Measurement of the $\mathrm{CO} 2$ apneic threshold in newborn infants: possible relevance for periodic breathing and apnea. J Appl Physiol 98:1171-1176

29. Clancy B, Darlington RB, Finlay BL 2001 Translating developmental time across mammalian species. Neuroscience 105:7-17

30. Javaheri S 1999 A mechanism of central sleep apnea in patients with heart failure. N Engl J Med 341:949-954 